\title{
Research on wireless sensor networks tissue repair method for live detecting of substation equipment
}

\author{
Hai $\mathrm{Yu}^{1}$, Lin Peng ${ }^{1}, \mathrm{He} \mathrm{Wang}^{1}$ and Hui Deng ${ }^{1}$ and Chang-Fu Xu ${ }^{2}$ \\ 1 Global Energy Interconnection Research Institute, Nanjing, Jiangsu, China \\ 2Jiangsu Electric Power Company Research Institute, Nanjing, Jiangsu, China \\ E-mail:yuhai@geiri.sgcc.com.cn
}

\begin{abstract}
For wireless sensor network in substation equipment charged detection, repair, such as communication failure causes changes in node network error of high power consumption during faults, putting forward the working cycle of the adaptive tissue repair method and low power network by dynamically adjusting control packet monitoring duty ratio can effectively balance the power and speed of the network tissue repair.
\end{abstract}

Keywords: Electric Substation Equipment Inspection; Wireless Sensor Network; Network Tissue Repair.

\section{Introduction}

Based on the wireless sensor network, the field application of the charging detection device of the substation equipment can significantly improve the efficiency of the charged detection work, and it is the development trend of the live line detection technology. Because of the poor deployment environment and limited capacity of the nodes, reliability is a major challenge for wireless sensor network. In this paper, the reliability of wireless sensor network system is studied from the view of network organization repairing.

\section{Tissue Repair Method for Wireless Sensor Network}

Wireless sensor network organization error can be regarded as for a sensor node, due to communication interference or node failure and other reasons, there is no available uplink node. The network organization reliability can be enhanced by increasing the system redundancy, but a lot of industrial wireless sensor network system uses a very low redundancy to reduce the cost. At the same time, sensor nodes communication ability is weak, the harsh deployment environment, so the 
wireless sensor network network organization errors often occur frequently, the efficient network organization repair mechanism to ensure it is low-power node and uplink node available fast network rediscovered.

\subsection{Routing repair mechanism}

In self-organizing routing mechanism of the wireless sensor network, i.e Directional diffusion[1], DV/DRP[2], when the upper node cannot be used, the need for routing repair. The current route repair general for the high redundancy deployment scenario, namely around the nodes in addition to the original uplink node, there are still more standby node, the routing failure node will broadcast route request message to recover the data routing. If the whole network broadcast, it will obviously lead to a high communication overhead.

The routing repair mechanism is not suitable for the network organization repair in the low duty cycle wireless sensor networks. This is because the network organization error in wireless sensor network not only contains the routing error, but also contains the time synchronization error. At present, most of the wireless sensor networks adopt synchronous intermittent operation mode, but the clock between nodes can not always be consistent, so they need to exchange time synchronization information periodically to keep synchronization. When a node cannot use, both time synchronization will be due to clock drift and failure, network organization error node cannot predict uplink node in the listening state time. At this time, even if the parent node node error recovery, network organization is also unable to determine when to send the routing request message. And if the broadcast routing request message, it is not only a huge power consumption, and may interfere with the communication of other nodes.

\subsection{Network organization repair mechanism based on control packet monitoring}

FireWxNet[3] uses the periodic control packet broadcast the way to carry on the network organization. The function of sink node periodically in network broadcasting control packets, generally consists of a route discovery and time synchronization information etc.; when a sensor node receives a control packet, update their routing and time synchronization information and further down the radio. Based on the mechanism, FirewxNet the is completely different from the above route repair mechanism of network organization repair methods: such as a sensor for a period of time cannot receive the package control, continuous 
monitoring channel to capture the control packet, to repair the network organization. LL-MAC[4] also uses a similar mechanism.

However, there is a serious flaw in this mechanism: when the node is in the network organization, it will not work. As a result, this mechanism is very high power consumption, the network organization for a long time may lead to a long time, the battery is running out of energy. This mechanism is called continuous monitoring network organization repair mechanism.

\subsection{Summary}

The existing routing repair mechanism does not apply to the low duty cycle of the wireless sensor network system. And the existing wireless sensor network organization repair technology depends on the continuous control packet monitoring, repair power is too high, resulting in the occurrence of network organization error node battery energy easily run out. So we need to study the new low power network organization and repair methods, and effectively balance the power and speed of network organization repair.

\section{Work Cycle Adaptive Low Power Network Tissue Repairing Method}

\subsection{Network organization repair}

In wireless sensor networks, network organization error, in addition to the routing error, often also contains time synchronization error. So when the node loses the parent node, it will be very fast because it can not receive the time synchronization information and lose synchronization. In order to ensure the normal operation of the network after the recovery of the network, the network organization must repair the time synchronization between the router and the node.

In order to achieve efficient network organization, we must consider two kinds of network organization errors: communication failure and node failure. Communication failure is often caused by channel interference. Node failure is often caused by node hardware or software errors or even node loss. Communication failures are often temporary, but the node failure usually lasts for a long time, because node repair requires manual operation, such as battery or node replacement. However, wireless sensor networks are often deployed in harsh environments, and manual maintenance is often not timely. Therefore, network organization errors in wireless sensor networks can be divided into two types, which are short time and long time. 
In view of the above problems, an adaptive low power network organization repair method is designed. First research network organization repair node of the intermittent working mechanism, to reduce power consumption of node in the route repair and time synchronization; then, according to the types of errors in different network organization repair needs, by dynamically adjusting the nodes in network organization repair intermittent work period, balanced and effective repair power and repair speed.

\subsection{Algorithm design}

\subsection{1. system model}

In the modified model, the sensor nodes have two different states: normal state and state of repair. If a node has joined the network, it works in the normal state. When a node for a period of time can not be found.

Network, which will enter the state of repair. In the state of repair, the node will carry out the channel monitoring to capture the control packets. Once successfully captured to the control package, the node will return to the normal state. In order to reduce the power consumption in the state of repair, it should be made to carry out the intermittent operation of the node in the network organization.

\subsubsection{Intermittent work network organization repair}

In the intermittent operation of the network organization, the node will only listen to the network during the time of the activity, and in the rest of the time to sleep to save energy. Since the duty cycle of the network is constant, the method is called static scheme. In normal state, the activity time, the sleep time and the working period of the nodes are respectively expressed by $T_{a}, T_{s}$ and $T_{w}$. At the same time, the activity time, the sleep time and the work cycle are respectively expressed by $T_{r a}, T_{r s}$ and $T_{r w}$ in the repair state. $T_{r a}$ must be different from $T_{a}$, because the node in the repair state may be due to the time synchronization error and can not know the control packet broadcast time. Since the control packet broadcast may occur at any time on $T_{w}, T_{r a}$ must be greater than or equal to $T_{w}$ ensure that the node is able to capture the control packets within the active time. For the consideration of energy saving, the general use $T_{r a}$ equal to $T_{w}$. 
$T_{r w}$ must be longer than $T_{r a}$ to save energy. However, this will cause the node to miss the control packets and lead to a slower rate of repair. Defines the time spent on the control packet listener to repair the delay, as defined in the control packet radio. The mathematical expectation of repair delay $A_{R}$ (mean repair delay) can be used by Eq. (1), $\lambda$ is $\left[\frac{T_{r W}}{T_{r a}}\right]$.

$$
\begin{aligned}
& A_{R}=\frac{T_{r a}}{T_{r W}} \sum_{i=1}^{\lambda-1} i T_{r a}+\left(1-\lambda \frac{T_{r a}}{T_{r W}}\right) \lambda T_{r a} \\
& =\lambda T_{r a}-\frac{\left(\lambda^{2}+\lambda\right) T_{r a}^{2}}{2 T_{r W}}=f_{a r}\left(T_{r a}, T_{r W}\right)
\end{aligned}
$$

\subsection{Dynamic intermittent work networks}

Although the static scheme can greatly reduce the power consumption of the node in the repair state, it does not distinguish between short time and long time network organization errors. In the static scenario, these two types of network organization errors have the same average repair delay and power consumption characteristics. This is not appropriate, because in the short time network organization errors, should give priority to repair speed so that nodes can return to normal as soon as possible; and in long time network errors should be preferred power to ensure that nodes can survive as long as possible. In order to solve this problem, this paper put forward the dynamic duty cycle adjustment mechanism, the mechanism makes the nodes in a state of repair the duty ratio is adjustable.

In the dynamic scheme, when the node is in the state of repair, it will first assume that this is due to the temporary communication failure caused by the use of a shorter. At this point, the nodes will have more frequent network monitoring to achieve the high speed of network organization repair. If the network can not be detected, the node will gradually increase $T_{r w}$ to reduce power consumption according to the Eq. (2).

$T_{r w(i)}=\left\{\begin{array}{cc}n^{i} T_{m}, & \text { if } \mathrm{n}^{i} T_{m}<T_{\max } \\ T_{\max }, & \text { else }\end{array}\right.$ 


\section{Performance Test Plan}

In this section, we will verify the performance by the theoretical analysis and the actual test of the wireless sensor network test bed. The nodes in wireless sensor network test bed are used to develop a wireless sensor network node, which is very similar to the MicaZ node. The theoretical analysis is based on the characteristics of the node. This section will be the first to test the performance in hair growth time network organization errors, and then test the performance in the short time network error has occurred, with the better performance in dealing with different types of network organization error analysis. In the test, the normal state of the work cycle is set to 1 minutes.

When an error occurs in a long time network, the power consumption of the network organization repair mechanism is preferred. In In the test, short time network organization errors is defined as the control packet broadcast will be restored in the adjustment phase, i.g $0<T_{\text {fail }} \leq T_{\text {tuning }}$, because $T_{\text {tuning }}$ should than most short time network organization errors continued for a long time to ensure these errors can quickly in the stage of adjustment to be repaired. In the test, the average repair time delay is used to evaluate the repair speed. At the same time, the repair efficiency is used to evaluate the performance of the network system in a short time. $F_{r}$ is determined by the additional repair energy consumption $E_{r}$ and $A_{r}$ as shown in the Eq. (3). Where $E_{r}$ represents the additional energy overhead caused by the repair delay.

$$
F_{x}=\frac{1}{E_{x} A_{x}}
$$

\section{Conclusion}

In this chapter, we study the reliability of wireless sensor network system in the network level, in two aspects of network organization reliability and data transmission reliability. In order to overcome the disadvantages of high energy consumption in the existing network organization repair mechanism, a new method of low power consumption network organization with adaptive operation cycle is proposed. Through the intermittent work in the repair of network organization, and dynamically adjust the working cycle, balance the power consumption and repair speed, so as to achieve the low power consumption of the network to ensure the reliability of the organization. The experimental results show that the battery life of the nodes can be greatly extended when the network is repaired. In addition, the error duration of network organization is relatively 
short, and the repair speed can be greatly improved by dynamic intermittent work cycle adjustment mechanism.

\section{Acknowledgment}

Thanks for the support of the science and technology project to State Grid Corporation "Research and pilot application of power grid equipment state intelligent sensing technology based on Internet of things".

\section{References}

1. Intanagonwiwat C, Govindan R Estrin D. Directed diffusion: a scalable and robust communication paradigm for sensor networks. Proceedings of the 6th Annual International Conference on Mobile Computing and Networking, 2000.

2. Hall C E Carzaniga A, Wolf A L. Dv/drp: a content-based networking protocol for sensor networks. Technical report, University of Lugano, 2006.

3. Hartung C, Han K Seielstad C. et a1. Firewxnet: a multi-tiered portable wireless system for monitoring weather conditions in wildland fire environments. Proceedings of the 4th Intemational Conference on Mobile Systems, Applications and Services, 2006.

4. Marin I, Arias J, Arceredillo E, et a1. LL-MAC: a low latency MAC protocol for wireless self-organised networks. Microprocessors and Microsystems, 2008, 32(4): 1 97-209. 